\title{
Tangence
}

\section{À propos de la création d'un lieu de sociabilité littéraire institué}

Analyse des débats et des enjeux qui ont précédé la création de l'Académie royale de langue et littérature françaises de Belgique

\section{On the Creation of an Established Venue of Literary Sociability} An Analysis of the Debates and Issues that Preceded the Creation of the Académie royale de langue et littérature françaises de Belgique

\section{Björn-Olav Dozo}

Numéro 80, hiver 2006

Sociabilités imaginées : représentations et enjeux sociaux

URI : https://id.erudit.org/iderudit/013546ar

DOI : https://doi.org/10.7202/013546ar

Aller au sommaire du numéro

Éditeur(s)

Tangence

ISSN

1189-4563 (imprimé)

1710-0305 (numérique)

Découvrir la revue

Citer cet article

Dozo, B.-O. (2006). À propos de la création d'un lieu de sociabilité littéraire institué : analyse des débats et des enjeux qui ont précédé la création de l'Académie royale de langue et littérature françaises de Belgique. Tangence, (80), 59-84. https://doi.org/10.7202/013546ar

\section{Résumé de l'article}

Les débats qui ont précédé la création d’une académie littéraire de langue française en Belgique ont duré vingt-cinq ans et s'articulent selon deux grands axes : l'un qualifié d'« institutionnel », l'autre d'« identitaire ». L'axe institutionnel renvoie au degré d'institutionnalisation que souhaite l'écrivain pour la littérature belge. L'axe identitaire permet de situer sa conception de l'indépendance de la littérature belge par rapport à la littérature française. On peut isoler six thèmes argumentatifs mobilisés lors des débats. Leur analyse met en évidence les enjeux de la création d'une académie, qui vont au-delà des représentations liées à un mode de sociabilité d'écrivains et engagent toute une conception de la littérature produite en Belgique francophone. 


\section{À propos de la création d'un lieu de sociabilité littéraire institué. Analyse des débats et des enjeux qui ont précédé la création de l'Académie royale de langue et littérature françaises de Belgique}

Björn-Olav Dozo,

Université de Liège-ARC Communauté française de Belgique

Les débats qui ont précédé la création d'une académie littéraire de langue française en Belgique ont duré vingt-cinq ans et s'articulent selon deux grands axes: l'un qualifié d'«institutionnel», l'autre d'«identitaire». L'axe institutionnel renvoie au degré d'institutionnalisation que souhaite l'écrivain pour la littérature belge. L'axe identitaire permet de situer sa conception de l'indépendance de la littérature belge par rapport à la littérature française. On peut isoler six thèmes argumentatifs mobilisés lors des débats. Leur analyse met en évidence les enjeux de la création d'une académie, qui vont au-delà des représentations liées à un mode de sociabilité d'écrivains et engagent toute une conception de la littérature produite en Belgique francophone.

Par-delà la question du type de sociabilité imaginée et réclamée par les écrivains belges à la fin du XIX siècle et des représentations que celle-ci engendre, nous souhaiterions attirer l'attention sur les enjeux qui existent derrière la volonté de créer un lieu de sociabilité propre aux littérateurs de Belgique. La création d'une académie de langue et littérature françaises dans ce pays n'est pas allée de soi, et les littérateurs ont mis du temps pour s'accorder à une large majorité sur les modalités de reconnaissance et de légitimation de la littérature francophone belge. Le mode de sociabilité littéraire qu'est l'académie, très codé et trouvant ses 
racines dans la vie littéraire française $\mathrm{du}_{\mathrm{XVII}}^{\mathrm{e}}$ siècle, ne s'est pas imposé comme une évidence aux écrivains belges. Les débats qui en précédèrent la création permirent dès le départ d'esquisser un nombre défini de "prises de position", que les agents, en fonction de leur "position» et de l'évolution de celle-ci, allaient revendiquer ou contester.

Notre objectif est de montrer comment, dans les discours, le modèle académique s'est mis en place et quelles furent les conséquences de la création d'une académie sur la vie littéraire en Belgique francophone. Nous nous focaliserons sur une analyse du discours émanant du monde littéraire (écrivains et critiques). Cette analyse nécessite une prise en compte des différentes réalités du pays: les clivages politiques et linguistiques bien sûr, mais surtout la prégnance du modèle français dans l'espace littéraire francophone. Suit donc une description de l'"espace discursif des possibles ", créé par les arguments des littérateurs belges qui réclamaient ou refusaient une institution académique appelée à structurer le champ littéraire belge francophone. La solution qui a émergé des argumentaires fut l'Académie royale de langue et littérature françaises de Belgique (en abrégé ARLLF), fondée par arrêté royal le 19 août 1920 . Â cette date et sur la proposition de Jules Destrée, ministre des Arts et des Sciences, le Roi concrétisait le souhait, controversé et longuement discuté, d'une partie importante des écrivains belges francophones.

\section{Outil méthodologique d'analyse du discours}

Arrêtons-nous d'abord sur les enjeux du débat, car ce n'est pas seulement une divergence de vue sur un mode de sociabilité propre aux littérateurs qui s'y exprime. Ce mode de sociabilité engendre des représentations de la littérature elle-même, et ce sont des conceptions très opposées de la littérature que manifestent les diverses prises de position sur l'opportunité de créer ou non une académie de littérateurs en Belgique francophone. Il est important de rappeler que, depuis la création de la Belgique, l'un des enjeux principaux des discours sur la production littéraire en Belgique francophone porte précisément sur l'existence même d'une littérature spécifiquement belge francophone. En effet, pour le dire très platement, s'il existe bel et bien en Belgique francophone une production littéraire de langue française, cette production a toujours connu des problèmes de dénomination. "Littérature belge de langue française», "littérature française de Belgique», "littérature 
francophone belge", autant d'appellations qui correspondent à des moments de son histoire. Mais les débats sur sa dénomination recouvrent, en fait, des questions qui mettent en cause son existence même: une frontière nationale suffit-elle à différencier la production littéraire belge de la production littéraire française? ou, a contrario, la communauté linguistique de deux littératures nationales suffit-elle à les rapprocher? Ces questions, résumées de manière abrupte, ont suscité une multitude de réponses au cours de l'histoire belge. Elles ont également trouvé de multiples lieux d'incarnation, et les débats qui ont précédé la création de l'Académie royale de langue et littérature françaises de Belgique en constituent un excellent exemple. En effet, avant de discuter la meilleure modalité de légitimation et de consécration pour une hypothétique littérature belge, certains ont repris la question de plus haut et placé l'existence même de cette littérature au centre du débat. D'autres, s'ils ne remettent pas en cause cette existence, s'interrogent néanmoins sur cette institution de la vie littéraire qu'est une académie et sur la conception de la littérature qu'elle engendre.

Voilà pourquoi, pour rendre compte de l'espace discursif des possibles dans sa complexité, il ne faut pas se contenter de penser la création d'une académie de littérature en Belgique francophone sur un seul axe, en fonction des avis énoncés soit par les tenants d'une structuration plus forte de la vie littéraire (les pro-institutions), soit par les opposants à toute forme d'institutionnalisation de la littérature, synonyme pour eux d'une mainmise du politique sur la littérature ${ }^{1}$. Ce premier axe discursif, dont nous venons d'esquisser les pôles, permet de situer la représentation que se fait l'écrivain du rapport entre la littérature et la sphère du pouvoir, notamment en ce qui concerne le rôle du politique dans l'institutionnalisation des lettres.

1. Certains agents considèrent en effet que la création par l'État d'une instance de consécration de la littérature belge serait un signe notoire du peu d'autonomie de cette littérature en regard de la sphère du pouvoir. Ils refusent ainsi toute institution de la littérature soutenue par l'État, par peur de compromettre un processus d'autonomisation du littéraire encore fragile, qui dépend en grande partie de la constitution d'un champ intellectuel balbutiant et que l'on ne peut aligner sur le modèle français. Sur l'histoire des intellectuels belges, voir Paul Aron et Marnix Beyen, "L'histoire des intellectuels en Belgique. Spécificités locales et actualité de la recherche", dans Michel Leymarie et Jean-François Sirinelli (sous la dir. de), L'histoire des intellectuels aujourd'hui, Paris, Presses universitaires de France, 2003, p. 409-425. 
Toutefois, pour comprendre les débats qui ont précédé la création de l'ARLLF, on ne peut se contenter de ce premier axe, l' «axe institutionnel». En effet, il faut ajouter un deuxième axe, qui permet d'estimer la représentation qu'a l'agent de l'indépendance de la littérature belge par rapport à la littérature française, voire par rapport au modèle français. Les différents intervenants prennent ce qu'ils jugent pertinents dans ce modèle pour défendre leur point de vue, qui peut être favorable ou non à la création d'une académie de littérateurs belges. Cette utilisation du modèle, en se prêtant à diverses inflexions, complexifie encore les possibilités de prises de position. Sur ce deuxième axe, l'«axe identitaire », deux pôles discursifs sont à isoler: du côté du premier, l'agent conçoit la production littéraire belge francophone comme trouvant pleinement sa place dans le champ littéraire français; l'existence d'un champ littéraire belge est niée et il n'existe aucune homologie à la frontière politique dans le champ littéraire. Du côté du second pôle discursif, l'agent adhère à la conception d'un champ littéraire belge ne subissant aucune domination du champ littéraire français. La position intermédiaire sur l'axe peut se décliner de diverses manières, lesquelles supposent toutes, cependant, que l'agent reconnaît un rapport de dominant à dominé entre le champ littéraire français et le champ littéraire belge, mais maintient la distinction entre les deux. Les différentes modalités de cette prise de position reposent sur l'emprunt au modèle français de divers attributs (son autonomie, son modèle institutionnel, ses écoles littéraires, etc.) dont l'agent dote la littérature francophone de Belgique selon la représentation qu'il a de celle-ci.

Cet outil méthodologique permet de dégager des tendances générales dans le discours de l'agent: cependant, chaque axe ne peut se concevoir que comme un continuum, et il n'y a pas de frontière nette entre deux prises de position.

L'outil descriptif mis en place permet notamment de dégager plusieurs zones ou «lieux de discours». On obtient alors le schéma suivant : 
Axe identitaire

«Litt. belge dépendante de la France»

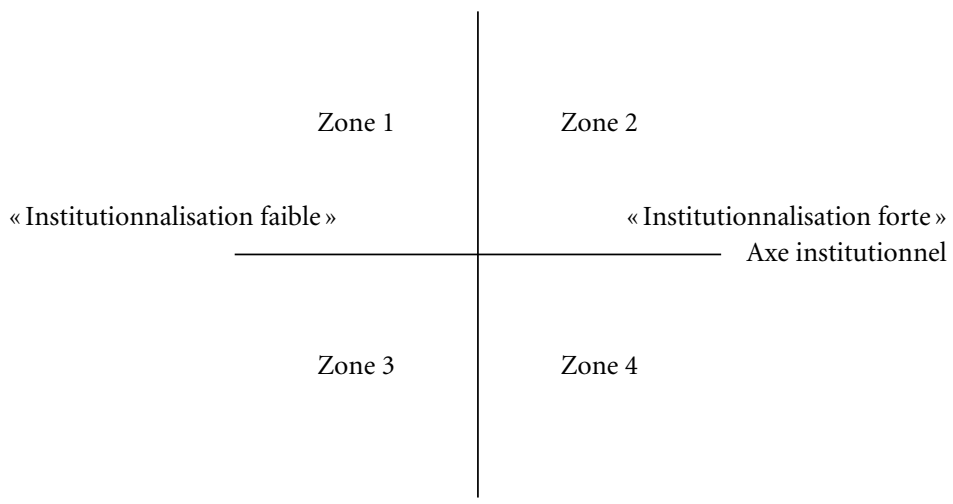

«Litt. belge indépendante de la France»

Schéma qui peut se retraduire par une matrice à double entrée:

\begin{tabular}{l|c|c} 
& «institutionnalisation faible» & «institutionnalisation forte» \\
\hline $\begin{array}{l}\text { «litt. belge } \\
\text { dépendante } \\
\text { de la France» }\end{array}$ & Zone 1 & Zone 2 \\
\hline $\begin{array}{l}\text { «litt. belge } \\
\text { indépendante } \\
\text { de la France» }\end{array}$ & Zone 3 & Zone 4 \\
\hline
\end{tabular}

Ces «lieux de discours» sont constitués de thèmes argumentatifs en nombre fini, que nous allons passer en revue en les illustrant. Il faut garder à l'esprit que chaque agent, par sa prise de position spécifique, se réapproprie le noyau sémantique d'un ou plusieurs de ces thèmes argumentatifs, qu'il reformule en fonction de sa position.

Ces thèmes argumentatifs mêlent représentations concernant soit le lieu de sociabilité en question, soit la littérature produite en Belgique. Le corpus qui concerne ces débats s'étend sur une période 
de vingt-cinq ans allant de 1897 à $1922^{2}$. Malgré cette durée, les thèmes argumentatifs restent assez constants, avec des résurgences, à différents moments, en fonction de l'actualité et du contexte d'énonciation, de l'un ou l'autre thème. Il faut souligner également que le même thème a pu être utilisé pour soutenir des avis contraires. C'est ainsi que des personnes en complet désaccord (par exemple un agent prônant une institutionnalisation forte de la littérature belge, indépendante de la France, et un autre soutenant une dépendance totale de la littérature belge à l'égard de la littérature française) vont utiliser le même thème argumentatif, mais en renversant les arguments.

\section{Les thèmes argumentatifs}

\subsection{L'enquête de Durendal}

Le moment fondateur du débat sur l'institutionnalisation de la littérature belge est une enquête réalisée auprès des gens de lettres. Il est possible de décrire tous les thèmes argumentatifs qui seront mobilisés au fil des débats à partir de cette première enquête publiée sur le sujet et parue dans la revue Durendal en avril $1899^{3}$.

Avant cette date, quelques articles épars avaient réclamé l'incorporation de littérateurs dans l'Académie royale de Belgique. Cette académie de savants, fondée en 1769 par Marie-Thérèse, donc bien avant la création en 1830, de la Belgique, accueillait au départ des "hommes de lettres» en vue d'encourager les sciences et les arts. Appelée communément la "Thérésienne», cette académie avait néanmoins refusé, en 1898, de recevoir de nouveaux littérateurs en son sein. La génération montante, celle de La jeune Belgique et des différentes revues ayant émergé en même temps qu'elle, ne pouvait donc prétendre à une reconnaissance officielle. De plus, l'opposition entre une jeunesse montante et les "perruques» de l'Académie, symbolisées par Charles Potvin, dernier littérateur accepté à la Thérésienne, n’était pas nouvelle.

2. Le corpus a été balisé une première fois par Denis Janowski, "Études des débats qui ont précédé et commenté la création de l'Académie royale de Langue et Littérature françaises (1883-1921) », mémoire de licence, Bruxelles, Université Libre de Bruxelles, 1994-1995, sous la direction de Paul Aron; puis une seconde fois par Raymond Trousson, Petite histoire de l'Académie, Bruxelles, Académie royale de langue et de littérature françaises, 1999.

3. «Une Académie de Littérateurs», Durendal, Bruxelles, avril 1899, p. 304-324, et la réponse d'Edmond De Bruijn dans le numéro suivant, p. 432-436. 
C'est à la suite de ce refus d'incorporation de nouveaux écrivains que Durendal publie son enquête, en sollicitant l'avis des principaux littérateurs du pays. Durendal les interroge sur l'opportunité et les modalités de création d'un organe de consécration pour les littérateurs. Les réponses constituent un ensemble de textes qui offrent la possibilité d'isoler un corpus homogène en synchronie, répondant au même contexte d'énonciation et permettant, en raison du statut de la revue Durendal à l'époque, d'embrasser une part représentative du champ littéraire belge francophone. De fait, à cette occasion, la revue n'interroge pas seulement ses collaborateurs habituels, ce qui évite de fausser l'échantillon en faveur d'une trop forte composante catholique, et sa légitimité lui garantit des réponses.

Six thèmes argumentatifs utiles pour l'analyse peuvent être dégagés des réponses à cette enquête. Tous sont des arguments concernant l'institutionnalisation des lettres belges. Cependant, tous sont marqués du sceau de l'identitaire et tous font intervenir une représentation de la littérature belge dans son rapport avec la littérature française.

Les six thèmes argumentatifs sont les suivants: l'utilité et le rôle des académies, le traitement égalitaire des langues en Belgique, le rôle qu'une académie permettrait à l'État de jouer dans la promotion des lettres belges, les conséquences de la création d'une académie sur l'originalité du mouvement belge, le travail de l'écrivain et la grandeur du pays.

\subsection{De l'utilité et du rôle des académies}

\section{Plus qu'un lieu de sociabilité: une institution}

Avant d'en venir au premier thème, qui touche à l'utilité et au rôle des académies, il faut s'interroger sur le mode de sociabilité réclamé dans les débats. Comme on l'a vu, la création d'une académie n'était pas la seule possibilité envisageable. Certains, dans l'enquête de Durendal, remettent explicitement en cause le mode de sociabilité choisi : Demolder, par exemple, prône une ligue - celle-ci assumant pourtant les mêmes fonctions qu'une académie, ce qui le rend dès lors favorable à une institution de la vie littéraire. Mais on s'aperçoit rapidement que ce type de débat sur le mode de sociabilité n'est finalement qu'une étiquette destinée à désigner un autre objet, qu'un redoublement de la problématique concernant la conception même de la littérature: à vrai dire, on 
reporte sous la rubrique du mode de sociabilité les tensions qui existent entre des conceptions de la littérature différentes. Les intervenants eux-mêmes le savent bien: le débat autour du nom du lieu de sociabilité est un faux débat car il ne constitue pas le fond du problème. On le voit bien à la lecture de ce passage du «Vœu des écrivains ", lettre adressée à Jules de Trooz, ministre de l'Intérieur et de l'Instruction publique, par l'Association des écrivains belges à la suite d'une enquête détaillée ayant eu 49 répondants:

Êtes-vous, comme nous, partisans [...] [de] la création, sous la forme de Chambre de Rhétorique, d'Académie de littérature française à l'instar de l'Académie flamande, ou Classe nouvelle ajoutée à l'Académie de Belgique, d'une Commission littéraire permanente quelconque, reconnue par l'État, autonome, jouissant de la personnalité civile et d'un budget spécial, et ayant pour but la culture et le développement des Lettres françaises en Belgique $^{4}$ [?]

Ainsi, les appellations se valent. Seule compte la volonté de s'engager dans un projet visant à instituer la vie littéraire, peu importe qu'il s'agisse d'une académie exclusivement consacrée à la littérature ou d'une classe des lettres rattachée à la Thérésienne.

Malgré tout, au plus fort de la controverse, quand les positions se sont cristallisées, le débat sur l'appellation porte en lui l'opposition entre des conceptions littéraires divergentes: des enjeux de pouvoir symbolique se dissimulent alors derrière le nom que les agents revendiquent pour l'organe à créer. Par exemple, Wilmotte, en 1906, veut réduire l'institutionnalisation des lettres belges à une sorte de syndicat - il est question d'un « organisme professionnel régulièrement constitué et reconnu par l'État, chargé de centraliser les subsides ${ }^{5} »$. Rency lui reproche alors de se contenter d'un organe qui se chargerait du "partage de la caisse ${ }^{6} »$, sans pouvoir symbolique, honorifique et de consécration d'aucune sorte. Deux conceptions s'affrontent, que l'on peut situer sur l'axe institu-

4. Alex Pasquier, «L'Académie belge et les rapports entre les écrivains et l'État", Nos Lettres. Association des écrivains belges de langue française, Bruxelles, vol. XXIII, $\mathrm{n}^{\text {os }}$ 9-12, septembre-décembre 1959, p. 18-21; vol. XXXIV, ${ }^{\text {os }} 4-6$, avril-juin 1960, p. 15-18; vol. XXXV, ${ }^{\text {os }} 7-10$, juillet-octobre 1960, p. 11-16.

5. Voir Maurice Wilmotte, Godefroid Kurth et al., "Rapport de la Classe des lettres à M. le Ministre», Bulletins de la classe des Lettres et des Sciences morales et politiques et de la classe des Beaux-Arts, Bruxelles, 1906, p. 352, où il est question d'" un organisme professionnel régulièrement constitué et reconnu par l'État [qui soit] chargé de centraliser les subsides».

6. Georges Rency, «Échos», Le Samedi, Bruxelles, 25 août 1906, p. 11. 
tionnel: l'une à tendance autonomisante, l'autre institutionalisante. La première, dans sa conception, rejoint l'avis de Séverin dans l'enquête de Durendal:

Je ne vois nullement la nécessité de créer au sein de l'Académie Royale une classe spécialement réservée aux Belles-Lettres. Cette création ne nous vaudrait, selon toute probabilité, ni une belle page de prose ni deux beaux vers de plus. Alors, à quoi bon?

S'agit-il de récompenser le mérite de tel ou tel écrivain? Encore une fois, à quoi bon? Quand on peut écrire Hors du Siècle, ou les Miroirs de Jeunesse, ou Entrevisions, par exemple, on trouve sa récompense en soi-même ${ }^{7}$.

Séverin explicite une représentation de la création littéraire qui correspond à une conception autonomisante de la littérature: elle s'inspire du modèle français qui s'est constitué au cours du $\mathrm{XIX}^{\mathrm{e}}$ siècle sous le paradigme de la Modernité et qui a détaché la littérature du modèle académique en affirmant son autonomie. Or, l'intervention de l'État, qui passe par le pouvoir politique et la consultation des "savants» (les philologues de la Thérésienne), remet en cause cette autonomie.

En soi, l'argument ne permet pas de trancher si l'agent conçoit la dépendance du champ littéraire belge par rapport au champ français. Il indique seulement qu'il a intégré l'idée d'autonomie du champ littéraire que postule le modèle français. En effet, le champ littéraire français fonctionne, si l'on en juge par les avis exprimés dans Durendal, soit comme recours - lors d'une stratégie d'entrisme, c'est-à-dire quand un littérateur belge part à Paris chercher une légitimité ou une reconnaissance, car il conçoit la France comme sa patrie littéraire -, soit comme modèle, lorsque l'écrivain a une représentation de la littérature qui correspond au modèle autonomisant à la française. Séverin semble adopter l'idée d'un champ littéraire à la française, conçu comme autonome, non soumis aux académies et sur lequel le champ littéraire belge doit s'aligner (les trois œuvres qu'il cite sont d'ailleurs d'auteurs belges, respectivement d'Albert Giraud, de Louis Delattre et de Charles Van Lerberghe).

Ce premier thème argumentatif permet, en somme, de situer l'avis des littérateurs uniquement en fonction de l'axe institutionnel. Mais, bien souvent, la question concerne aussi l'utilité et le rôle d'une académie spécifique à la Belgique francophone, ce qui

7. Fernand Séverin, Durendal, art. cité, p. 318. 
permet au thème d'être beaucoup plus marqué sur le plan identitaire.

\section{De l'utilité et du rôle des académies en Belgique francophone}

Dans l'enquête de Durendal, les intervenants comparent souvent les rôles de la future académie de littérateurs belges et ceux de l'Académie française. Cette comparaison permet d'évoquer les deux grandes missions assignées à ces institutions: l'une d'ordre linguistique, l'autre artistique. Les autres missions (attribution de prix, d'encouragements, etc.) ne sont pas traitées comme des mandats spécifiques d'une académie: elles concernent les encouragements financiers à la littérature, encouragements qui ne passent pas nécessairement par une académie, une commission consultative ou de gestion pouvant suffire à remplir cet office. On le voit, le débat sur les prix et les encouragements recouvre les prises de position qui départagent les tenants de l'autonomie et ceux de l'institution, et que l'on retrouve par exemple entre Wilmotte et Rency. Or, comme Rency le sent bien, une académie doit se fixer d'autres buts que l'attribution de prix et la distribution d'encouragements (bien qu'il reconnaisse qu'il s'agit tout de même d'une de ses missions). Quant à la mission linguistique, elle consiste principalement en une action: sauvegarder le beau et le bien dire ou, comme le dira Liebrecht dans Le Thyrse en 1906, "avoir une influence morale, épurer la langue, encourager et développer sa culture $^{8} »$. Certains y verront ainsi un double emploi par rapport à l'Académie française; d'autres, au contraire, une nécessité pour le champ littéraire belge.

Dans l'enquête de Durendal, on retrouve surtout l'argument de double emploi par rapport à l'Académie française. Ainsi, seul Fierens-Gevaert prend explicitement position en faveur de la création d'un équivalent de l'Académie française pour la Belgique. Il insiste de plus sur les tâches attribuées à cette nouvelle académie, tâches qui sont l'exact équivalent de celles assumées par l'Académie française:

[elle] s'appliquerait à combattre les wallonismes et les flandricismes, chercherait en outre s'il n'y a pas lieu d'enrichir le voca-

8. Henri Liebrecht, "L'Académie et les Littérateurs ", Le Thyrse, Bruxelles, $1^{\mathrm{er}}$ août 1906, p. 101. Nous soulignons.

9. Hippolyte Fierens-Gevaert, Durendal, art. cité, p. 313-314. 
bulaire français de certains mots du parler belge, très harmonieux, très expressifs, et qui méritent à tous égards d'être introduits dans la langue écrite ${ }^{9}$.

Cet argument mettant en évidence le rôle linguistique d'une académie correspond à un modèle puriste nuancé, soucieux d'enrichir le patrimoine linguistique français en prenant en compte l'apport de la Belgique.

La mission artistique, on l'a vu avec l'avis de Séverin, est ramenée la plupart du temps à l'utilité d'une institution capable d'assurer la qualité de la production littéraire. Il ne s'agit pas d'une réflexion concernant le cas de la Belgique, mais l'utilité des académies en général. Quand il est marqué au coin de l'identitaire, le thème argumentatif de la mission artistique d'une institution encadrant la vie littéraire sert surtout aux opposants d'une académie belge: plusieurs en refusent la création, dénonçant son inutilité au regard des fonctions déjà dévolues à l'Académie française. Ainsi, Georges Eekhoud fonde sa conception du champ littéraire sur l'argument linguistique:

Avec M. Godefroid Kurth je doute fort qu'on rende un vrai service à la littérature en enrégimentant ses principaux (?) représentants dans une Académie. L'Académie française, à Paris, suffit amplement. Il n'y a qu'une langue française. Alors pourquoi deux académies ${ }^{10}$ ?

Cette réfutation de l'utilité littéraire d'une académie en Belgique, du fait de l'identité des langues entre la Belgique et la France, dénote une conception du champ littéraire fondée uniquement sur l'argument linguistique. La communauté de langue entre la France et la Belgique francophone pourrait apparaître comme un fait vérifiable, qui définirait un ensemble littéraire homogène de manière objective. Pourtant, il ne faut pas perdre de vue que l'idée qu'une littérature se définissant sur une base linguistique est une représentation: mentionnons la triade herderienne "nation - langue - littérature", conséquence bien connue de cette représentation, qui se trouve à la base du thème argumentatif du traitement égalitaire des langues par l'État belge.

10. Georges Eekhoud, Durendal, art. cité, p. 318. 


\subsection{Le traitement égalitaire des langues en Belgique}

Le deuxième thème argumentatif est celui du traitement égalitaire des langues en Belgique: l'exemple le plus caractéristique est le texte de Valère Gille. Reprenons sa réponse à l'enquête :

Vous avez vu plus haut que l'une et l'autre [les littératures française et flamande de Belgique] ont leur place réservée dans la Classe des Lettres. Or, le 8 juillet 1886, l'arrêté suivant figurait au Moniteur:

«Voulant donner une nouvelle preuve de Notre sollicitude pour les intérêts des lettres néerlandaises;

"Considérant que rien ne peut en favoriser davantage le développement que l'institution d'une Académie littéraire permanente;

»Sur la proposition de Notre Ministre...

» Nous avons arrêté et arrêtons:

» ARTICLE PREMIER. - Il est institué à Gand, sous la dénomination de «Koninklijke..., etc.», une Académie de littérateurs et de savants ayant pour objet l'étude et la culture de la langue et de la littérature néerlandaises.

ART. 4. - Il y a vingt-cinq membres titulaires; vingt de ces membres doivent être Belges et avoir leur résidence en Belgique; les cinq autres peuvent être choisis parmi les littérateurs et savants néerlandais domiciliés en Belgique.»

Ma réponse? Le principe d'égalité des langues exige que l'on fasse pour les Belges français ce qu'on a fait pour les Belges flamands. Que l'on prenne le même arrêté [celui de la création de l'académie flamande] en remplaçant le mot néerlandais par le mot français.

C'est une question de droit ${ }^{11}$.

Cet argument n'est concevable qu'en fonction d'une logique dualiste étendue à la littérature de Belgique conçue de ce fait comme une littérature nationale en deux langues. Les mesures de soutien apportées à la production littéraire en Belgique doivent être strictement les mêmes de part et d'autre de la frontière linguistique. On conçoit donc derrière cette exigence l'idée d'une forte indépendance du champ de production littéraire francophone belge, mis en concurrence directe avec le champ de production littéraire flamand. Le point de comparaison n'est plus, par conséquent, à

11. Valère Gille, Durendal, art. cité, p. 308. 
chercher du côté du modèle français, mais inscrit bien au contraire dans l'ensemble politique national connu comme étant la Belgique. Les mesures en faveur d'un groupe linguistique dans cet ensemble national doivent trouver un écho dans l'autre, ce qui incite à penser la littérature produite en Belgique comme largement indépendante de celle produite chez ses voisins linguistiques, les Pays-Bas ou la France.

À l'opposé de cette conception, mais utilisant le même argument, Maurice Dullaert affirme:

Et, tout d'abord, on ne saurait, se fondant sur l'égalité des langues en Belgique, conclure de l'existence d'une académie flamande à la création d'une académie des lettres françaises. L'Académie flamande a tout au moins une raison d'être philologique que n'aurait point l'autre académie : celle-ci n'usurperait pas sans ridicule une tutelle confiée dès longtemps aux Quarante par le cardinal de Richelieu ${ }^{12}$.

Ce «tout au moins» souligne le refus de recourir à l'argument de la parité pour justifier la fondation d'une Académie francophone. Sa conception du champ littéraire est fondée sur la langue et l'on ne saurait projeter les frontières politiques sur le champ littéraire: les lettres francophones belges font partie intégrante des lettres françaises.

Reste une troisième actualisation possible de cet argument de l'égalité des langues: une institution qui accueillerait les deux langues sans distinction. Seul à pousser la logique d'une littérature en deux langues aussi loin, Edmond De Bruijn soumet de la sorte le champ littéraire uniquement aux frontières politiques héritées d'une «tradition» :

L'Académie, ses publications administratives, les communications de ses Bulletins, devraient être ou pouvoir être bilingues et les membres de l'Académie flamande de Gand repris dans les Classes des Lettres et Beaux-Arts de la seule Académie. Outre que c'est réparer une disproportion et éviter un antagonisme, c'est se conformer au bon sens qui ne peut considérer l'emploi d'une langue comme certificat d'identité d'une tradition, c'est d'ailleurs faciliter les efforts vers une gloire nationale: car, Verhaeren, par exemple, où le voudrions-nous voir, à l'Académie flamande ou à l'Académie de Belgique ${ }^{13}$ ?

12. Maurice Dullaert, Durendal, art. cité, p. 319.

13. Edmond De Bruijn, Durendal, art. cité, p. 432-433. 
Une seule académie pour toute la Belgique: cette position sera très rare dans la suite des débats; elle découle d'une représentation unitaire de la Belgique en tant que nation, qui, selon le mythe romantique diffusé par Herder, se doit de posséder, si ce n'est une langue (commune), du moins une littérature unique.

\subsection{Rôle que confère une académie à l'État dans la promotion des lettres belges}

Le troisième thème argumentatif est le rôle de l'État dans la promotion des lettres belges. Hippolyte Fierens-Gevaert développe longuement la relation bénéfique que pourraient entretenir l'État et la littérature:

Après vingt années d'efforts persévérants, les littérateurs français de notre pays n'ont pas encore réussi à conquérir l'attention du public belge. Les trop rares encouragements officiels qu'on leur a distribués ne pouvaient les dédommager de l'indifférence de leurs compatriotes. La nouvelle génération, moins féconde et moins enthousiaste que la précédente, semble souffrir d'une sorte de lassitude, de découragement. Les aînés, en général, n’ont réussi qu'à s'assurer des situations matérielles médiocres; [...].

Nos compatriotes attachent une grande importance aux consécrations officielles. L'écrivain, l'artiste ou le savant qui n'occupe pas quelque poste important ou qui n'est point décoré n'est rien à leurs yeux. On le traite, suivant l'expression de Balzac, de «zéro social». Le bénéfice moral que nos écrivains retireraient de leur entrée à l'Académie serait considérable. Jouissant enfin d'un prestige officiel égal à celui des autres membres: savants, musiciens, peintres, etc., ils prendraient une importance soudaine aux yeux de la foule. Grandis dans l'opinion générale, ils concevraient plus nettement leur rôle qui est, non de se confiner dans un idéal égoïste - ainsi que l'ont fait plusieurs d'entre eux - mais de populariser leur rêve, d'enseigner à tous la beauté. Nos écrivains ne devraient-ils pas une grande reconnaissance au gouvernement qui, par son initiative, aurait accompli ce miracle de rendre les lettres à leur vraie mission éducatrice? Et ainsi se réconcilieraient dans notre pays les classes dirigeantes et les hommes de pensée dont l'isolement actuel peut entraîner de déplorables conséquences. La création d'un "corps littéraire » sera donc utile aux écrivains d'abord, ensuite au pays lui-même ${ }^{14}$.

14. Hippolyte Fierens-Gevaert, Durendal, art. cité, p. 312-313. 
Beaucoup de points seraient à considérer dans cet extrait. On se contentera de souligner les plus intéressants dans la perspective qui nous occupe. Tout d'abord, l'argument en lui-même lie de manière forte la littérature à la politique: il postule que la littérature a un rôle d'illustration nationale, et qu'à l'inverse, le pouvoir politique peut avoir une influence sur la diffusion de la littérature. Or, ce postulat définit de la sorte une césure entre la production littéraire belge (quelle que soit sa langue) et les autres littératures. En particulier, il établit une différence entre la production littéraire francophone belge et la production littéraire française. On peut donc en conclure, en fonction de l'axe identitaire, que l'argument affirme une certaine forme d'indépendance de la littérature francophone belge par rapport au champ de production français, conçu comme une littérature nationale.

Ensuite, Fierens-Gevaert repousse explicitement le confinement du poète dans un "idéal égoïste». Il répond de la sorte à l'argumentation de la Thérésienne, qui voyait le poète comme individu, créateur pour lui-même. Fierens-Gevaert défend une conception du poète comme étant l'émanation d'une collectivité, diffuseur des valeurs de celle-ci et pourvu d'une mission éducative vis-à-vis de la «foule». On retrouve ici l'opposition entre l'individu et la collectivité, à laquelle correspondent deux logiques littéraires différentes. Enfin, Fierens-Gevaert émet le souhait que soient distribuées des sinécures à certains écrivains. Ces places de fonctionnaire de l'État réservées à une certaine catégorie de personnel littéraire (Fierens-Gevaert parle des «aînés qui n'ont réussi à s'assurer que des situations matérielles médiocres » et pour lesquels l'État devrait faire quelque chose) font donc partie des arguments utilisés par les partisans d'une académie. L'utilisation qu'il fait de ce troisième thème argumentatif assimile les lettres belges à un ensemble autonome différenciable dans le champ littéraire francophone et authentifie l'existence à part entière de cette littérature en lui conférant les attributs d'une «littérature nationale».

En évoquant la même problématique du rôle de l'État, Georges Marlow arrive à des conclusions totalement opposées:

Qu'Albert Giraud, Valère Gille, Fernand Séverin ou Maurice Maeterlinck enjolivent la couverture de leurs volumes de la vignette du futur institut ou de la firme de leur librairie, ils seront toujours de merveilleux poètes admirés des lettrés et ignorés du public. Ce n'est pas la sanction gouvernementale qui 
forcera le peuple à acheter leurs livres, et ceux qui les lisent n'ont pas besoin de leur consécration officielle pour les aimer ${ }^{15}$.

Autrement dit, Marlow juge que l'État n'a pas de rôle à jouer dans la promotion des lettres et, de ce fait, sa conception de la littérature est très différente de celle défendue par Fierens-Gevaert. "Ignorés du public» ou encore "ce n'est pas la sanction gouvernementale qui forcera le peuple à acheter leurs livres » : voilà autant de syntagmes qui dénotent une conception de la littérature comme sphère de production restreinte, loin de ces préoccupations éducatives envers la collectivité que doit nourrir le littérateur selon Fierens-Gevaert. Marlow ne nie pourtant pas la spécificité d'un ensemble littéraire belge (tous les noms qu'il utilise comme exemples sont belges), mais le modèle français de la sphère de production restreinte conditionne son approche de la littérature francophone belge:

Il me paraît qu'en réunissant à date fixe nos artistes en une salle austère pour y ébaucher, à l'instar des académiciens de Paris, quelque vague dictionnaire, où le mot «vers» sera prétexte à querelles entre Iwan Gilkin et Émile Verhaeren, on ne fera pas même la fortune d'un éditeur ${ }^{16}$.

Le syntagme "à l'instar de Paris » souligne le caractère français du modèle académique tel qu'on envisage de l'importer en Belgique francophone. La future académie, aux yeux de Marlow, ne sera jamais qu'un calque de l'institution française.

\subsection{Conséquences sur l'originalité du mouvement belge}

Le quatrième thème argumentatif examine les conséquences de la création d'une académie sur l'originalité du mouvement belge. Cet argument, qui postule l'existence d'un mouvement belge reconnaissable et identifiable comme tel, est le plus immédiatement identitaire. Il présuppose un mouvement littéraire belge que rendent clairement identifiable ses caractéristiques propres: jeunesse, fougue, liberté et «mœurs barbares», comme l'écrit Des Ombiaux. Cette représentation constitue une prise de position identitaire explicite: la littérature belge existe et seules les conséquences de son institutionnalisation servent d'arguments pour mieux soutenir ou refuser une académie.

15. Georges Marlow, Durendal, art. cité, p. 309.

16. Georges Marlow, Durendal, art. cité, p. 309. 
Les avis à propos de ces conséquences sont, là encore, partagés. Louis Delattre, par exemple, voit dans cette originalité une qualité qui ne saurait s'épanouir qu'en dehors de toute institutionnalisation:

Une si petite fille que notre littérature on la voudrait déjà serrer du corset académique et "procuster» du brodequin de l'officiel? - Non, n'est-ce pas, vous ne le laisserez pas faire?

Songez qu'en 1635, où Richelieu prit fantaisie de réunir ses hommes de lettres, Villon, Rabelais, Ronsard et Montaigne, étaient morts de longtemps. Quatre siècles de production littéraire continue avaient formé une tradition française. Et c'était une solide commère, capable de se défendre, que le cardinal livrait aux entreprises des Gombauld, des Conrart, des Lamothe et autres immortels inconnus...

Encore le commerce de ces vieux messieurs anémia-t-il si singulièrement la gente dame que, longtemps, elle passa pour mourante!

Ah! laissons donc courir notre petite fille à sa fantaisie. Durant quelques siècles encore, laissons-la mûrir au loisir du bon soleil libre.

Nous, nous n'y serons plus. Mais j'ose parier, allez, qu'il se présentera bien, et plus tôt que nécessaire, quelque compagnie de podagres pour enclore, dès qu'elle sera nubile, notre littérature aux gages, et la contraindre aux tristes jeux des dictionnaires, des palmes et des couronnes ${ }^{17}$ !

Delattre utilise la jeunesse de la littérature belge pour filer une métaphore sur sa résistance à une institutionnalisation trop précoce. En la comparant avec la France, il met en évidence deux ensembles littéraires, le français et le belge, et utilise pour sa démonstration l'image du «raccourci» opéré par la littérature belge face au modèle français, laquelle rappelle ainsi la longue période de temps dont ont bénéficié la langue et la littérature françaises avant d'être épurées et rationalisées.

Maurice Des Ombiaux, à l'opposé, aspire à un meilleur contrôle de cette "originalité » belge grâce à la future académie. En effet, la particularité qu'il reconnaît aux lettres belges le gêne:

Créer une classe destinée aux littérateurs, n'est pas nécessairement enrégimenter ceux-ci. Le talent de nos écrivains

17. Louis Delattre, Durendal, art. cité, p. 317. 
n'augmentera pas de leur entrée dans une Académie, mais il est tout aussi certain qu'il n'en sera pas diminué.

Cette légère divergence exprimée, je suis, comme M. Kurth, d'avis que l'utilité d'une telle section n'est pas péremptoire pour nos hommes de lettres. Cependant ne croyez-vous pas que notre littérature ne pourrait que gagner, si ses principaux représentants se rencontraient assez assidûment dans ce salon qu'est, avant tout, une Académie? Elle y perdrait de sa... comment dirais-je, de sa sauvagerie, si vous voulez, quoique le terme soit un peu vif. Cette littérature nationale ne date pas de longtemps, elle sort des ténèbres et comme toute l'humanité en sa jeunesse, elle a des mœurs assez barbares. L'Académie adoucirait peut-être ces mœurs, ce qui, à tout prendre, serait déjà un heureux résultat ${ }^{18}$.

À partir du même argument identitaire, on voit donc deux intervenants émettre un avis profondément différent sur le sens d'une institution du littéraire, l'un jugeant ce dernier profitable, l'autre reconnaissant le bienfait de sa disparition. Chacun emprunte au modèle français: Delattre se réclame d'une autonomie du littéraire, telle qu'elle s'illustre dans la France de la fin du XIX siècle, loin des institutions, tandis que Des Ombiaux souhaite parfaire la littérature belge en la "polissant» sur le modèle classique français.

\subsection{Travail de l'écrivain}

Le cinquième thème argumentatif concerne la représentation de l'écrivain, perçu généralement comme «solitaire et inspiré». Il est important de souligner à quel point cette représentation est redevable à la figure du poète romantique, prophète enthousiaste touché par la grâce. Ici, le poète est conçu comme détaché de toute contingence matérielle, loin des académies et des titres honorifiques, autonome par rapport à la sphère du pouvoir. Pascale Casanova a montré que cette image du poète autonome trouve son point de cristallisation à Paris, ville qui «dénationalise, départicularise, littérarise» les textes:

Le champ littéraire français étant le plus "avancé» dans

l'émergence de ce phénomène deviendra ainsi à la fois un modèle et un recours pour les écrivains de tous les autres champs qui aspirent à l'autonomie ${ }^{19}$.

18. Maurice Des Ombiaux, Durendal, art. cité, p. 315.

19. Pascale Casanova, La république mondiale des lettres, Paris, Seuil, 1999, p. 126. 
Cet argument est utilisé par la Thérésienne pour refuser l'élection de nouveaux littérateurs en son sein. En effet, suivant son jugement, le travail du savant s'effectue en groupe, là où le travail de l'écrivain en est un de solitaire. Il est intéressant de noter comment les intervenants se réapproprient l'argument dans leurs réponses, certains le citant même, comme le fait Eekhoud:

Comme l'a fort bien dit le professeur Kurth, il est de l'essence du travail artistique de devoir ses chefs-d'œuvre à la méditation solitaire et à l'inspiration personnelle. La même autorité universitaire a clairement défini le rôle de la classe des lettres actuelle de l'Académie de Belgique. Elle a sa raison d'être comme classe des sciences. Les écrivains d'imagination, les créateurs proprement dits, poètes, conteurs, dramaturges, n'y seraient point à leur place. Ils y perdraient même leur temps ${ }^{20}$.

D'autres l'allient à l'originalité du mouvement belge pour en faire une propriété définitoire de l'écrivain belge. Cette représentation de la littérature nationale, que définiraient certains traits caractéristiques, est couplée à la représentation de «l'écrivain solitaire et inspiré». Cette dernière est donc considérée comme définitoire, comme l'assure Maurice Dullaert:

Les lettres sont nées, ont grandi et fleuri, chez nous, spontanément, librement, sauvagement, hors et loin de toutes académies. Pourquoi changerions-nous le régime dont elles se trouvent si bien? Comme le dit excellemment M. Godefroid Kurth: «Il est de la nature du travail scientifique de progresser dans les ateliers communs et grâce aux efforts collectifs; il est de l'essence du travail artistique de devoir ses plus beaux chefs-d'œuvre à la méditation solitaire et à l'inspiration personnelle ${ }^{21}$."

Dullaert se réclame de Kurth. Pourtant, à y regarder de près, jamais dans son argumentaire Kurth ne parle de "notre» littérature, ou ne manifeste le moindre signe dénotant une prise de position en faveur de l'existence d'une littérature belge. Dullaert ne peut donc utiliser cet argument de "l'écrivain solitaire et inspiré » pour défendre une littérature belge que par une particularisation de la portée de cette représentation, particularisation qui va à l'encontre de l'image généralement admise et qui s'est imposée au cours du $\mathrm{XIX}^{\mathrm{e}}$ siècle $^{22}$ au cour même du processus d'autonomisation: c'est à

20. Georges Eekhoud, Durendal, art. cité, p. 318.

21. Maurice Dullaert, Durendal, art. cité, p. 321.

22. Comme le défend Pascale Casanova dans La république mondiale des lettres, ouv. cité. 
Paris et en rapport au champ littéraire français que l'écrivain acquiert sa plus grande indépendance. Dullaert s'approprie donc l'argument de Kurth en en faisant une propriété définitoire du littérateur belge : celui-ci se tient naturellement à l'écart des institutions académiques et les lettres belges ont grandi «spontanément, librement, sauvagement hors et loin de toutes académies ». Dullaert a intériorisé le modèle français, mais l'adapte à la Belgique en le «naturalisant».

\subsection{Pays trop petit, production trop restreinte, régionale}

Le sixième thème argumentatif renvoie à la représentation du pays, à sa grandeur et à ses capacités en termes de production littéraire. Il est à remarquer que, dans l'enquête de Durendal et, par la suite, dans le corpus de documents rassemblés autour des débats sur la création de l'Académie, cette représentation n'est utilisée comme argument que lorsqu'elle est négative ${ }^{23}$. Elle sert donc à rejeter le projet d'académie, le pays étant trop petit pour la pourvoir en nombre suffisant d'hommes de valeur. Maeterlinck, par exemple, ironise:

Je ne sais s'il est bien nécessaire que soit fondée une Académie Belge. Mais enfin, si quelques esprits le désirent, soit. Il y a, m'assure-t-on, plus d'une académie régionale, de ce genre: Auvergnate, Bretonne, Gasconne, etc. Elles sont en général obscures, muettes et inoffensives ${ }^{24}$.

Si une telle approche consiste à réduire la littérature belge à une segmentation régionale de la littérature française, une variante nuance quelque peu ce point de vue en comparant les deux littératures - ce qui offre l'avantage de ne pas considérer le monde des lettres belges comme une «région française». Mais, la référence demeurant toujours le modèle français, dans un cas comme dans l'autre, la conclusion ne varie pas: la production française reste dominante.

Dans un pays où la production littéraire est forcément et restera toujours assez restreinte, prodiguer les palmes, ce serait non

23. Excepté à un moment, lors des discours célébrant la création de l'ARLLF, au sortir de la guerre, alors que les exploits de la Belgique sur le plan politique lui donnent la possibilité de revendiquer le fait d'être un "grand pays", si ce n'est par la taille, du moins par le mérite. Voir l'utilisation qu'en fait Destrée lors de l'inauguration de l'ARLLF.

24. Maurice Maeterlinck, Durendal, art. cité, p. 322. 
seulement en déprécier l'honneur, mais couronner souvent des médiocres et, par suite, se discréditer. Il ne faut pas que les distinctions académiques deviennent une forme d'aumône, ni que l'académie se confonde avec l'assistance publique ${ }^{25}$.

Bref, au nom de l'argument identitaire, demandons-nous, avant de créer des institutions, ce qu'elles pourraient bien instituer... Cette conception de la littérature, proche de celle du Manifeste $d u$ Lundi $i^{26}$, exprime un penchant autonomisant important: dans un champ autonome (perçu comme un espace de concurrence), seuls les meilleurs émergent. L'intervention d'une instance extérieure au champ (ici l'État, dont l'action est décriée par la comparaison avec l' "assistance publique») en perturbe le jeu normal. Dullaert reprend donc encore une fois le modèle autonomisant français, qu'il adapte à la représentation qu'il se fait des réalités belges: «production restreinte» et, de ce fait, «à trop vouloir honorer, on honorerait les médiocres»).

L'analyse des thèmes argumentatifs permet ainsi une distribution des prises de position des agents en fonction des lieux de discours qu'a permis de dégager notre outil méthodologique. Tous sont utilisés pour défendre ou attaquer l'idée d'une académie, sauf celui de la grandeur du pays. Il faudra attendre après la Première Guerre mondiale pour qu'on y recoure positivement et qu'il serve d'argument en faveur de la création d'une académie ${ }^{27}$.

\section{Analyse des «lieux de discours»}

La combinaison de tous ces thèmes argumentatifs permet de dégager des "lieux de discours» en fonction des deux axes présentés plus haut. Comme on l'a vu, la position de l'agent dans tel «lieu de discours» est fonction de son utilisation du thème

25. Maurice Dullaert, Durendal, art. cité, p. 320.

26. Pour une analyse socio-rhétorique des enjeux du Manifeste du Lundi, voir Jean-Marie Klinkenberg, "Lectures du "Manifeste du groupe du lundi" (1937) ", Lettres de Belgique. En hommage à Robert Frickx, Cologne, Janus Verlagsgesellschaft, 1992, p. 98-124.

27. Pour un exemple de cet usage de l'argument, voir Björn-Olav Dozo, «Le héros dans La bataille littéraire après la Grande Guerre: une représentation au service d'une littérature nationale belge", @nalyses. Revue de critique et de théorie littéraire, numéro intitulé Portrait de l'homme de lettres en héros, Collectifs, Écrivains hérö̈ques du long XIX é siècle, 2006-03-09. http://www.revue-analyses.org/document.php?id=101. 
argumentatif. Pour répartir les agents énonciateurs, il faut se livrer à une analyse de chaque thème, utilisé pro ou contra, en rapport avec les axes présentés plus haut et de manière à placer chaque agent dans l'une des zones définies.

Il faut garder à l'esprit que chaque thème argumentatif doit être considéré comme un indice, un indicateur d'une tendance générale. La présence de plusieurs indicateurs permet de confirmer l'orientation privilégiée dans une réponse par rapport à la problématique identitaire. Mais il ne faut jamais perdre de vue que ce que l'on repère, ce sont les jalons identitaires d'un discours portant sur une question institutionnelle, avec une interprétation de ces indices qui permet de dégager une tendance vers l'un ou l'autre pôle de l'axe identitaire. Il est toutefois impossible de déterminer de manière univoque la conception que se fait un agent du champ littéraire belge, comme on peut le faire pour sa conception de l'institutionnalisation de la littérature à partir de l'enquête de Durendal. À vrai dire, il aurait fallu que l'enquête pose explicitement la question de l'indépendance ou de la dépendance du champ littéraire belge par rapport au champ français, car l'implication identitaire des thèmes argumentatifs utilisés dans cette enquête se situe à un niveau différent de la question de l'institutionnalisation proprement dite de la littérature.

Si l'on croise les indices identitaires avec la réponse à propos de la création d'une académie, on obtient le tableau suivant:

\begin{tabular}{|l|l|l|}
\hline & «institutionnalisation faible » & «institutionnalisation forte \\
\hline $\begin{array}{l}\text { «litt. belge } \\
\text { dépendante } \\
\text { de la France }\end{array}$ & $\begin{array}{l}\text { Zone 1 } \\
\text { Godefroid Kurth; Georges } \\
\text { Marlow; Georges Eekhoud; } \\
\text { Maurice Maeterlinck; Charles } \\
\text { Van Lerberghe }\end{array}$ & \multicolumn{1}{|c|}{ Zone 2 } \\
\hline $\begin{array}{l}\text { «litt. belge } \\
\text { indépendante } \\
\text { de la France } »\end{array}$ & $\begin{array}{l}\text { Zone 3 } \\
\text { Edmond Picard; Louis Delattre; } ; \\
\text { Séverin; Maurice Dullaert; } \\
\text { Maurice Cartuyvels; José } \\
\text { Hennebicq; Camille Lemonnier }\end{array}$ & $\begin{array}{l}\text { Valère Gille; Eugène Demolder; } \\
\text { Iwan Gilkin; Arnold Goffin; } \\
\text { Hippolyte Fierens-Gevaert; } \\
\text { Maurice Des Ombiaux; Hector } \\
\text { Hoornaert; Émile Verhaeren; } \\
\text { Georges Rency; Edmond } \\
\text { De Bruijn }\end{array}$ \\
\hline pas tranché & Henri Maubel (pas d'argument) & \\
\hline
\end{tabular}


Il faut signaler trois cas particuliers pour cette enquête de Durendal: Giraud, qui déclare ne pas répondre aux enquêtes, Maubel, qui n'emploie pas d'argument, et Eugène Demolder. Celui-ci s'oppose explicitement à la constitution d'une académie, mais soutient la création d'une ligue, qui «propagera la lecture des auteurs belges ${ }^{28} »$. Il réclame donc, malgré tout — suivant l'analyse faite plus haut du devenir des débats sur la nature de l'instance institutionnalisant la littérature —, une institutionnalisation plus forte de la littérature belge, et est à sa place dans la quatrième zone, dans le coin inférieur droit du tableau.

À la lecture de ce tableau, une évidence s'impose: en 1899, aucun des agents ne tient un discours réclamant une forte institutionnalisation tout en revendiquant l'appartenance de la littérature belge à la littérature française (Zone 2). Ce possible discursif n'est pas actualisé. Les deux pôles les plus importants qui se dégagent de ce classement sont ceux qui couplent d'une part « institutionnalisation forte» et «indépendance du champ littéraire français» (Zone 4), d'autre part «institutionnalisation faible» et «dépendance du champ littéraire " (Zone 1).

Quelques années après l'enquête de Durendal, ces deux pôles vont cristalliser toutes les prises de position après le «Vœu des écrivains", et les discours autour de la création ou non d'une académie ne seront jamais aussi marqués par la question de l'identité de l'écrivain belge francophone. Au plus fort des débats, aucune prise de position intermédiaire n'est possible: si l'on est contre l'institutionnalisation de la vie littéraire belge, on soutient la dépendance du champ littéraire belge par rapport au champ français. Durant la période de précipitation (au sens chimique) des débats, la confusion entre l'institutionnel et l'identitaire est donc totale, et c'est véritablement la reconnaissance d'un champ littéraire belge qui est en jeu, de manière directe et non nuancée: son institutionnalisation à la faveur d'un mode de sociabilité spécifique est alors le garant de son existence.

Le troisième pôle, qui privilégie une faible institutionnalisation d'un champ littéraire belge indépendant, regroupe des agents ayant pour la plupart tiré des conséquences négatives de l'originalité du mouvement belge. Ce mouvement, pour eux, n'est pas partie intégrante du champ français, mais bien signe

28. Eugène Demolder, Durendal, art. cité, p. 307. 
d'indépendance de la Belgique par rapport à la France du point de vue littéraire. Autonome, il est défini généralement comme une nature. Celle-ci est en fait l'actualisation en Belgique du modèle français de champ littéraire autonome, retraduit et "naturalisé », car ce modèle possède un ancrage français très fort: Paris reste la capitale de l'autonomie littéraire ${ }^{29}$ et, par conséquent, l'unique moyen pour le champ littéraire belge d'actualiser ce modèle est de faire disparaître cette composante française de l'autonomie en en faisant une «nature» propre à la littérature belge.

\section{La fondation de l'ARLLF, ou l'actualisation du dernier « lieu de discours" virtuel}

Lors de la fondation de l'ARLLF, plusieurs éléments seront privilégiés dans le discours d'inauguration. Leur combinaison va permettre d'actualiser le dernier possible discursif: une littérature dépendante du champ littéraire français, mais bénéficiant d'une institutionnalisation forte. Ce dernier possible discursif induit donc une représentation de la littérature produite en Belgique francophone spécifique au lieu de sociabilité particulier qu'est l'ARLLF. Voyons maintenant quels éléments discursifs et factuels permettent cette représentation.

Les deux premiers éléments proviennent du critère de sélection des premiers académiciens: seront membres fondateurs de l'ARLLF les lauréats des prix quinquennaux (roman et poésie) et triennaux (théâtre). Outre le fait que ce critère ne sera pas respecté complètement ${ }^{30}$, il induit deux conséquences: il assure la « reproduction» d'un corps d'élite par le choix d'agents déjà consacrés ${ }^{31}$ qui éliront à leur tour ceux qui, à leurs yeux, seront «méritants»; et il correspond, par les genres que les prix consacrent, à une conception de la littérature qui prend appui sur le modèle français classique. Les

29. Voir Pascale Casanova, La république mondiale des lettres, ouvr. cité.

30. Voir Paul Aron, "Questions académiques. 1920-1940», Textyles, Esneux (Belgique), n 15, 1998, p. 136, qui met en évidence l'éviction de deux auteurs de théâtre de boulevard, jugés assurément trop peu dignes des lauriers académiques...

31. Treize des vingt premiers académiciens ont publié dans La Jeune Belgique; l'histoire littéraire traditionnelle les lie fortement au mouvement prenant naissance au début des années 1880, qu'elle consacre généralement comme la «renaissance des lettres belges». La conception de la littérature de ces agents — un modèle littéraire parisien adapté aux réalités belges — a donc marqué indubitablement le discours d'inauguration de l'ARLLF. 
genres nobles (poésie et théâtre) ou plus récemment valorisés (roman) sont en effet mis à l'honneur.

D'autres éléments discursifs renforcent cette conception de la littérature: comme l'a souligné Paul Aron ${ }^{32}$, le discours de Destrée et le règlement d'ordre intérieur de l'académie permettent de cerner la pratique littéraire qui sera soutenue par l'ARLLF.

Les termes choisis orientent d'emblée la «littérature" vers des pratiques se référant au classicisme du Grand Siècle ( Défense et illustration...»), inscrites dans une hiérarchie des genres («les œuvres les plus hautes») et dans une vision toute scolaire de l'écriture ("art d'écrire... éveiller le goût... propager le respect»). L'objet ainsi valorisé est également précisé: il s'agit bien des "lettres françaises", formule à laquelle l'Académie restera d'ailleurs fidèle, et qui inscrit la production locale dans l'espace dominé par le centre éditorial et culturel parisien ${ }^{33}$.

D'autres discours d'inauguration vont dans ce sens, notamment celui de Maurice Wilmotte ${ }^{34}$.

S’ajoute à ces éléments discursifs un élément factuel, qui inscrit de manière nette la représentation de la littérature qu'ont les créateurs de l'ARLLF dans une logique propre au champ littéraire français: la création de dix sièges (sur quarante) pour des membres étrangers. Par la création de ces sièges, Destrée opère une adaptation du modèle français: il reprend les prérogatives qui sont traditionnellement dévolues à Paris par sa domination linguistique (rassemblement de la francophonie autour d'un centre) et les transpose à la Belgique. Sa justification n'est plus d'ordre linguistique (comme l'est celle de Paris, capitale de la langue française), mais d'ordre politique (le prestige de la Belgique lors des épreuves de la guerre). On retrouve l'argument de la dimension du pays, mais utilisé positivement pour la première fois. Destrée reprend du modèle français l'aspect institutionnalisant du champ littéraire par rapport à la francophonie dont la Belgique assumerait, dans ce contexte, le rôle de carrefour ${ }^{35}$.

Si l'on résume les différents éléments discursifs et factuels, on peut dégager une position qui est propre à l'ARLLF : en effet,

32. Paul Aron, «Questions académiques. 1920-1940», art. cité, p. 7.

33. Paul Aron, "Questions académiques. 1920-1940", art. cité, p. 7.

34. "Séance d'installation. Discours de M. Maurice Wilmotte», Bulletin de l'ARLLF, Bruxelles, t. I, 1922, p. 46.

35. Franz Hellens utilisera aussi cet argument dans la revue moderniste qu'est Le Disque vert. Voir Franz Hellens, "La Belgique balcon sur l'Europe», Le Disque vert, Bruxelles, $\mathrm{n}^{\circ} 1$, novembre 1922, p. 34 . 
aucun des discours institutionnels et identitaires analysés jusqu'ici ne correspond à la position discursive mise en évidence pour l'ARLLF, qui défend une littérature belge fortement institutionnalisée et dépendante du champ littéraire français. Pour créer cette nouvelle position, l'Académie se fonde sur un compromis: elle utilise certains éléments institutionnels du modèle littéraire français (notamment la définition de son rôle comme lien entre les différents centres francophones), en ignore d'autres (la logique des écoles littéraires est complètement court-circuitée par l'hégémonie de La Jeune Belgique) et s'inscrit sous la domination littéraire du champ français. Mais cette subordination à l'hégémonie française est en fait un hommage rendu au passé de la France, à son époque classique et à son purisme linguistique: la hiérarchie des genres pratiqués par les agents élus en est une émanation.

Cette position nouvelle, occupant la seule «case vide» du schéma que nous proposons, ne représente donc pas la combinaison de toutes les conceptions de la littérature qui sont apparues au cours des débats - bien qu'il s'agisse néanmoins d'une forme de compromis entre les trois premières positions -, mais une attitude originale, qui définira la ligne de conduite de l'ARLLF au moins jusqu'au milieu des années $1960^{36}$. Elle se renforcera même avec les orientations que favorisera la troisième génération d'académiciens, signataires, pour certains, du Manifeste $d u$ Lundi.

Cette posture qu'adopte l'ARLLF dans l'espace discursif des possibles lui confère le rôle d'un acteur à part entière dans le champ littéraire belge, mais ne la désigne toutefois pas comme l'incarnation de la littérature belge. Ce qui devait au départ être le lieu de sociabilité du monde des lettres belge, du moins si l'on suit les conceptions imaginées par certains intervenants lors des premiers débats, est finalement devenu un acteur supplémentaire: non pas un lieu fédérateur, unifiant, représentatif de toutes les tendances de la production en Belgique francophone, mais le lieu de sociabilité d'une certaine frange des acteurs du champ littéraire belge.

36. En effet, quarante-quatre ans plus tard, l'ARLLF, lors d'une de ses séances mensuelles, décide de demander au Ministre de l'Éducation nationale de rebaptiser les cours d'Histoire de la littérature belge de langue française en Histoire de la littérature française de Belgique, et de les rendre obligatoires dans les programmes de cours de philologie romane (voir "Chronique», Bulletin de l'ARLLF, Bruxelles, t. XLII, 1964, p. 237). Cette décision s'inscrit dans la logique d'une institution belge soumise aux canons classiques français et à une conception d'un champ littéraire français passant outre aux frontières politiques. 